\title{
Quasi-periodic modulation of solar and stellar flaring emission by magnetohydrodynamic oscillations in a nearby loop
}

\author{
V. M. Nakariakov, C. Foullon, E. Verwichte, and N. P. Young
}

\author{
Physics Department, University of Warwick, Coventry, CV4 7AL, UK \\ e-mail: V.Nakariakov@warwick.ac.uk
}

Received 30 November 2005 / Accepted 20 February 2006

\section{ABSTRACT}

\begin{abstract}
We propose a new model for quasi-periodic modulation of solar and stellar flaring emission. Fast magnetoacoustic oscillations of a non-flaring loop can interact with a nearby flaring active region. This interaction occurs when part of the oscillation situated outside the loop reaches the regions of steep gradients in magnetic field within an active region and produces periodic variations of electric current density. The modulation depth of these variations is a few orders of magnitude greater than the amplitude of the driving oscillation. The variations of the current can induce current-driven plasma micro-instabilities and thus anomalous resistivity. This can periodically trigger magnetic reconnection, and hence acceleration of charged particles, producing quasi-periodic pulsations of X-ray, optical and radio emission at the arcade footpoints.
\end{abstract}

Key words. Sun: flares - Sun: oscillations - Sun: corona - stars: flare - stars: oscillations - stars: coronae

\section{Introduction}

Quasi-periodic pulsations (QPP) in radio, optical, X-ray emission generated in solar flares have been known for several decades (see, Aschwanden 2004; Nakariakov \& Verwichte 2005, for recent reviews). QPP with similar parameters have been detected in stellar flares (see, e.g. Mathioudakis et al. 2003; Mitra-Kraev et al. 2005, and references therein). Typical periods of QPP are in the range of a few $\mathrm{ms}$ to several min. This wide range of periods indicates that several different physical processes may be responsible for the generation of QPP. Periods longer than a few seconds are associated with magnetohydrodynamic (MHD) oscillations of coronal loops (e.g. Rosenberg 1970; Zaitsev \& Stepanov 1975; Roberts et al. 1984; and the above cited reviews). More precisely, periodicities in this range coincide with the theoretical estimations of the transverse and longitudinal travel times of acoustic and fast (or Alfvén) waves in coronal loops. Recently, this interpretation has been supported by direct, spatially resolved observations of various modes of MHD oscillations in the EUV and radio bands. The observational discoveries include standing kink, sausage and longitudinal modes. Periods of the standing modes are determined by the ratios of the loop lengths and characteristic speeds of the modes. Also, shorter periods have been registered and shown to be associated with propagating MHD waves guided by loops (Roberts et al. 1984; Nakariakov et al. 2004).

Normally, the relative amplitudes of the coronal MHD waves observed directly in the EUV and in the visible light bands are less than ten percent. On the other hand, QPP often detected in the microwave and X-ray bands have much deeper modulation, sometimes of a hundred percent. A typical solar flare with QPP, observed in the hard X-ray band, is shown in Fig. 1, demonstrating a periodicity of a few min and deep modulation of the emission. A question arises as to whether X-ray and microwave QPP are of the same nature as the MHD waves and oscillations directly observed in solar coronal structures and, if the answer is positive, more questions follow, as to what is responsible for the observed deep modulation and as whether it is connected with the electron acceleration process.

There have been several mechanisms proposed for the explanation of the X-ray and microwave QPP. In particular, dynamic models of magnetic reconnection predict that the processes of tearing instability and coalescence of magnetic islands occur iteratively, leading to an intermittent or impulsive bursty energy release and particle acceleration (e.g., Kliem et al. 2000). Tajima et al. (1987) demonstrated the possibility of oscillatory regimes in coalescence of current carrying loops. However, MHD oscillations remain the most attractive paradigm for QPP, because of the simple geometrical mechanisms (resonant or dispersive) for the creation of the periodicity and because of the vast direct observational evidence of these oscillations in coronal structures.

Brown \& Hoyng (1975) developed a betatron mechanism for QPP: the electron acceleration happens in a slowly vibrating magnetic bottle and the spectrum of the non-thermal electrons varies according to the vibrations. Zaitsev \& Stepanov (1982) suggested a model for flaring QPP, based upon the modulation of the mirror ratio in a magnetic trap situated in the loop near the initial flare energy release by sausage oscillations. This would lead to the periodic escape of the particles from the trap downward toward the loop footpoints and the subsequent precipitation of the particles at the footpoints, causing QPP. Asai et al. (2001) proposed another acceleration modulation mechanism, based upon the periodic modulation of the distance between two slow shocks at the magnetic reconnection site by an MHD oscillation in a loop located under the site.

In some observed cases, it is possible to determine the spatial structure of QPP and confirm that it is indeed connected with an MHD mode of the flaring coronal structure (e.g., the global sausage mode observed by Nakariakov et al. 2003). However, in other cases, the geometrical sizes of $X$ ray and microwave loops are very different from that required for the explanation of QPP observed in them in terms of the 


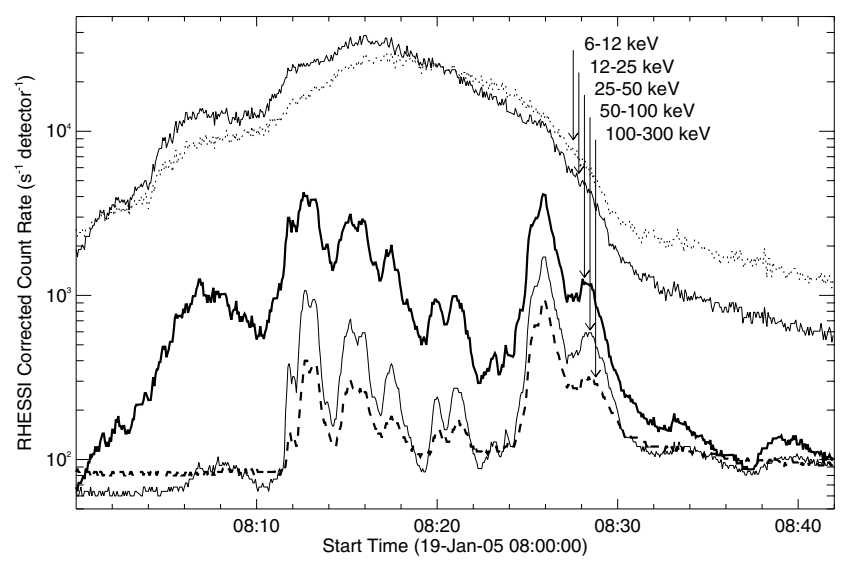

Fig. 1. A typical hard X-ray $(25-300 \mathrm{keV})$ solar flare with quasiperiodic pulsations, observed in full disk measurements by RHESSI on the 19th of January, 2005. The event is associated with a flare of class X1.3 (peaking at 08:22 UT, as seen by GOES $1-8 \AA$ ).

MHD oscillations. Recently, Foullon et al. (2005) analysed 10 min QPP in flares observed with RHESSI (Reuven Ramaty High Energy Spectroscopic Imager) and other instruments and suggested that, in the analysed case, QPP were likely to be caused by kink mode oscillations in a magnetically-linked large loop.

Here, we present a model which can explain the coupling of oscillations in nearby loops with QPP of flaring energy release. We suggest that periods of X-ray and microwave QPP observed in a flaring loop or at its footpoints are produced by MHD oscillations or propagating fast wave trains in another loop situated nearby, which is not necessarily magnetically linked with the flaring arcade. In the model, the MHD oscillations are not considered to be responsible for the flaring energy release itself, but play the role of periodic triggering, which modulates its efficiency. Thus, we separate the process and location of the flaring energy release on the one hand, from an MHD resonator which is responsible for the generation of the periodicity on the other hand.

\section{Generation of flaring QPP by external oscillation}

Coronal loops are known to support several MHD modes (e.g. Nakariakov \& Verwichte 2005): e.g. kink, sausage and longitudinal modes, which have different phase speeds, dispersion and symmetry of perturbations. As the side boundaries of the loop are open (the boundary conditions at the loop surface are the continuity of the perturbation of the total pressure and transverse displacement), MHD modes perturb the plasma not only inside the loop but also in the external medium. In leaky modes this external part of the mode propagates outside the loop, while the external part of trapped modes is evanescent. The penetration depth of a mode into the external medium is determined by physical parameters in the loop and by its period. For example, in a typical EUV loop (of length $200 \mathrm{Mm}$, with radius $1 \mathrm{Mm}$, Alfvén speed of $1000 \mathrm{~km} \mathrm{~s}^{-1}$ and density enhancement of a factor 2-3), the penetration depth of kink modes, both standing (with a period of about $6 \mathrm{~min}$ ) and propagating (with a period of about, say, $10 \mathrm{~s}$ ), is about $2 \mathrm{Mm}$. For sausage modes this value is even larger and growing when the longitudinal phase speed approaches the external Alfvén speed at a cut-off. These estimations demonstrate that an MHD mode of a loop can interact with other coronal structures which are situated at some distance from the oscillating loop.

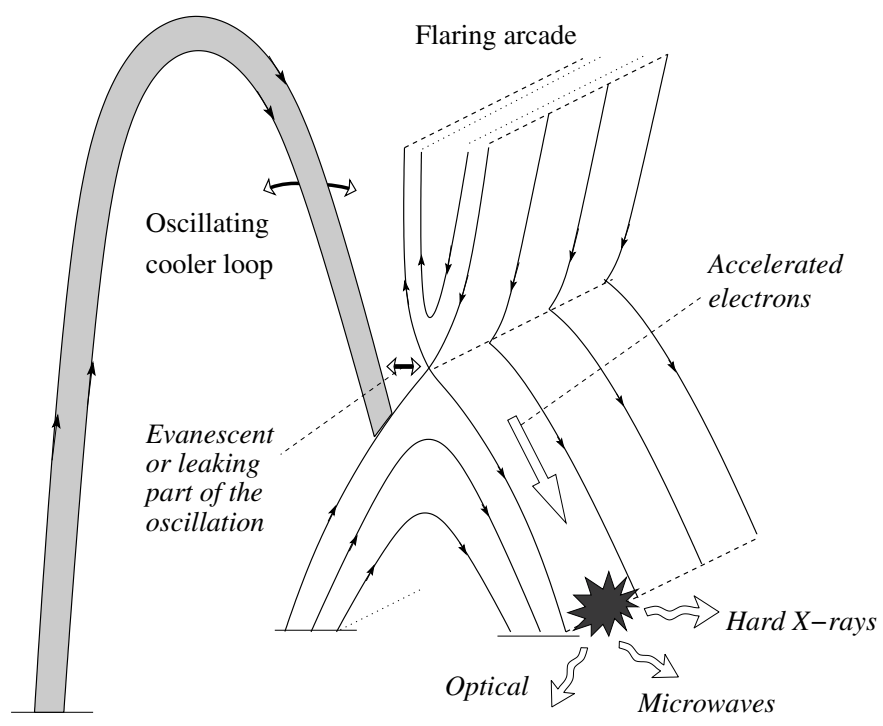

Fig. 2. A sketch of the mechanism for the generation of quasi-periodic pulsations of solar and stellar flaring emission. The cool (shaded) loop experiences fast magnetoacoustic oscillations (e.g., kink or sausage mode). A segment of the oscillating loop is situated nearby a flaring arcade. An external evanescent or leaking part of the oscillation can reach magnetic null points in the arcade, inducing quasi-periodic modulations of the electric current density. The resulting current driven plasma micro-instabilities are known to cause anomalous resistivity, which triggers magnetic reconnection. This periodically accelerates particles, which follow the field lines and precipitate in the dense atmosphere, causing quasi-periodic emission in radio, optical and X-ray bands.

In the following, we shall assume that there is a null-point magnetic non-uniformity near the oscillating loop (see Fig. 2), of much smaller size than the length of the loop. In this case the properties of MHD modes of the loop, as a global phenomenon, are still determined by the whole loop and are not significantly affected by the magnetic non-uniformity. The external part of transverse oscillations looks, from the point of view of the neutral point, as an incoming fast magnetoacoustic wave with the period prescribed by parameters of the oscillating loop. This effect can be produced by any fast mode - kink, sausage or flute as any of them produces a significant perturbation of the loop boundary.

Recently, McLaughlin \& Hood (2004, 2005) studied the interaction of an aperiodic fast magnetoacoustic pulse with two-dimensional single and double X-type neutral points and found that, in the vicinity of a neutral point, the pulse amplitude experiences amplification while its transverse wavelength significantly decreases. This leads to an increase of the electric current density associated with the pulse, which experiences an exponential growth up to a few orders of magnitude near the neutral point (see their Fig. 3).

Developing the studies of McLaughlin \& $\operatorname{Hood}(2004,2005)$ we have performed a simple 2D numerical simulation of the interaction of a periodic fast magnetoacoustic wave with a magnetic neutral point, with the use of Lare $2 \mathrm{~d}$ code (Arber et al. 2001). The plasma temperature is taken to be uniform, the plasma- $\beta$ is equal to 0.02 at the domain boundary. The normalising values are the magnetic field strength and the Alfvén speed at the domain boundary, and the characteristic spatial scale of the field inhomogeneity. A harmonic perturbation of the transverse component of the velocity is generated at the boundary of the computational domain. The induced fast magnetoacoustic wave propagates across the field, towards the neutral point. Without 


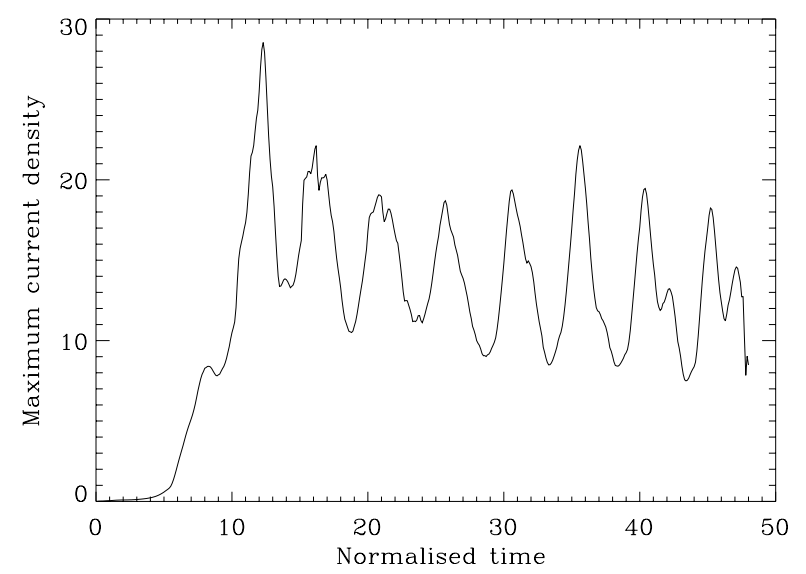

Fig. 3. Time evolution of the maximum electric current density in the vicinity of a $2 \mathrm{D}$ magnetic neutral point. The current is generated by a harmonic fast magnetoacoustic wave with period of 5 time units, coming towards the neutral point. The initial relative amplitude of the wave is $1.5 \%$. The plasma $\beta$ is finite. The normalising values are the magnetic field strength and the Alfvén speed at the domain boundary, and the characteristic spatial scale of the field inhomogeneity.

the wave, the magnetic configuration is force-free and the electric current is absent. The curl of the magnetic field perturbations in the fast wave induces an electric current. The decrease of the Alfvén speed in the vicinity of the neutral point leads to the decrease of the wavelength, increasing the current density. Because of the 2D structuring, the wave is refracted towards the neutral point and hence focussed. For a single fast pulse, this effect is described in detail by McLaughlin \& Hood (2004). Finite $\beta$ effects, included in our model, do not change this effect qualitatively. The non-uniformity in the Alfvén speed and in the components of the magnetic field causes a complicated pattern of wave interference. In the vicinity of the neutral point, there are steep gradients in the perturbations of the magnetic field and, consequently, a build-up of high electric current.

The time dependence of the maximum current density near the neutral point, observed in the simulations, is shown in Fig. 3 (cf. Fig. 1). The periodicity of the curve is prescribed by the driver, while the amplitude is much higher. For the parameters used in the simulation, the modulation depth reaches $30 \%$ for an initial wave amplitude of $1.5 \%$. The presence of a weak longitudinal magnetic field (perpendicular to the plane of the neutral point) does not qualitatively change this result. More detailed results on the fast wave interaction with a neutral point will be published elsewhere. Independent of these results, our simulations clearly demonstrate that the periodicity in the coming fast waves is efficiently transmitted into the periodic modulation of the current, which builds up along the separatrices.

\section{Discussion}

The energy of the incoming fast waves and hence the energy of the built-up current is tiny, less than the energy of a nanoflare (e.g., a loop segment of length $10 \mathrm{Mm}$ and radius $1 \mathrm{Mm}$, filled by a plasma with the concentration $10^{10} \mathrm{~cm}^{-3}$, oscillating with the amplitude $20 \mathrm{~km} \mathrm{~s}^{-1}$, has the energy of about $10^{24} \mathrm{erg}$ ). Thus, the periodic modulation of the current cannot on its own explain observed QPP. However, the electric current is known to induce efficiently plasma micro-instabilities of various kinds, and hence produce anomalous resistivity in the region of the current concentration. Since the characteristic times of micro-instabilities are normally shorter than the times of MHD processes (e.g.
Benz 2002), the generation of the anomalously high resistivity follows the variations of the current almost instantly. The anomalous resistivity is a popular ingredient of the fast magnetic reconnection theory, as it can efficiently trigger flaring energy release (e.g. Yokoyama \& Shibata 1994; see, also Priest \& Forbes 2000 for a review). Consequently, the periodic variation of the electric current at the vicinity of an X-point can cause periodic triggering of reconnection, producing QPP.

The proposed model links QPP in flaring light curves with fast magnetoacoustic oscillations in a coronal loop situated near the flare site. We have demonstrated that the linkage can be carried out by fast magnetoacoustic perturbations of the medium external to the oscillating loop, in the form of an evanescent or leaky part of the oscillating mode. Thus, QPP are prescribed by the period of the oscillation, which can have periods from a few seconds (in the case when the oscillation is caused by a propagating fast wave train) to several minutes (in the case of the global kink mode, cf. Foullon et al. 2005). For typical relative amplitudes of solar coronal oscillations, of a few percent, the interaction of the evanescent or leaky "tails" of the oscillations with a magnetic neutral point at the flare site is shown to produce quasi-periodic variations of the electric current density, with relative amplitudes reaching up to $30 \%$. When the current variations exceed the threshold of some current driven microinstability, the current-generated anomalous resistivity can trigger reconnection energy release. As the current is periodically modulated, this can generate quasi-periodic pulsations of electron acceleration. Hence, the "external" periodicity can be found in light curves of flaring emission produced by precipitating electrons. Here, we do not consider details of either the reconnection energy release or particle acceleration at all, restricting our attention to the ideal MHD part of the problem and to the qualitative discussion of the proposed mechanism only.

Recent spatially resolved observations (e.g. Grigis \& Benz 2005) demonstrate that the 3D structure of hard X-ray QPP is quite complicated. In particular, while the integral light curve contains clear QPP, different bursts (or maxima of the QPP) are coming from different loop footpoints of the flaring arcade. This finding can be easily explained by the proposed model. The fast magnetoacoustic wave front which triggers magnetic reconnection is necessarily affected by local inhomogeneities of the Alfvén speed. As a result, the wave front becomes non-planar, possibly even filamented, with one or several tongues stretched towards the regions of the decreased Alfvén speed - e.g. the null points. The fast wave can then reach only certain sites along the arcade. In turn, footpoints of these sites, which are distributed parallel to the axis of the arcade, become highlighted by the emission. The physical conditions in the part of the arcade affected by the triggered reconnection would not necessarily allow for another reconnection. But the next reconnection event may be triggered by the next wave period elsewhere along the arcade and will result in the observed spatial structure of footpoint flaring.

Here, we have discussed only one possible mechanism to link the incoming fast waves and the modulation of the flaring $\mathrm{X}$-ray and microwave emission: the periodic triggering of magnetic reconnection by a fast wave through the periodic switching on of the anomalous resistivity. The proposed mechanism does not exclude other possible mechanisms for the modulation. In particular, the fast wave can modulate the distance between the slow shocks in the model of Asai et al. (2001). Moreover, nonlinear steepening of the fast wave in the vicinity of a neutral-point (see, e.g. Landi et al. 2005) can provide an efficient electron acceleration mechanism without reconnection. 
Acknowledgements. We would like to thank T.D. Arber, B. Dennis, V.F. Melnikov and L. Ofman for helpful discussions, and E. Kontar for attracting our attention to the event on 19/01/2005. V.M.N. acknowledges support of a Royal Society Leverhulme Trust Senior Research Fellowship; V.M.N., E.V. and N.P.Y. acknowledge the support from PPARC.

\section{References}

Arber, T. D., Longbottom, A. W., Gerrard, C. L., \& Milne, A. M. 2001, J. Comp. Phys., 171, 151

Asai, A., et al. 2001, ApJ, 562, L103

Aschwanden, M. J. 2004, Physics of the Solar Corona (Berlin: Springer Praxis Books)

Benz, A. O. 2002, Plasma Astrophysics: Kinetic Processes in Solar and Stellar Coronae (Dordrecht: Kluwer)

Brown, J. C., \& Hoyng, P. 1975, ApJ, 200, 734

Foullon, C., Verwichte, E., Nakariakov, V. M., \& Fletcher, L. 2005, A\&A, 440, L59

Grigis, P. C., \& Benz, A. O. 2005, ApJ, 625, L143

Kliem, B., Karlický, M., \& Benz, A. O. 2000, A\&A, 360, 715
Landi, S., Velli, M., \& Einaudi, G. 2005, ApJ, 624, 392

Mathioudakis, M., Seiradakis, J. H., Williams, D. R., et al. 2003, A\&A, 403, 1101 Mitra-Kraev, U., Harra, L. K., Williams, D. R., \& Kraev, E. 2005, A\&A, 436, 1041 McLaughlin, J. A., \& Hood, A. W. 2004, A\&A, 420, 1129

McLaughlin, J. A., \& Hood, A. W. 2005, A\&A, 435, 313

Nakariakov, V. M., \& Verwichte, E. 2005, Living Rev. Solar Phys., 2, 3. URL(05/07/2005): http://www . livingreviews . org/lrsp-2005-3

Nakariakov, V. M., Melnikov, V. F., \& Reznikova, V. E. 2003, A\&A, 412, L7

Nakariakov, V. M., Arber, T. D., Ault, C. E., et al. 2004, MNRAS, 349, 705

Priest, E. R., \& Forbes, T. G. 2000, Magnetic Reconnection: MHD Theory and Applications (Cambridge: Cambridge Univ. Press)

Roberts, B., Edwin, P. M., \& Benz, A. O. 1984, ApJ, 279, 857

Rosenberg, H. 1970, A\&A, 9, 159

Tajima, T., Sakai, J., Nakajima, H., et al. 1987, ApJ, 321, 1031

Yokoyama, T., \& Shibata, K. 1994, ApJ, 436, L197

Zaitsev, V. V., \& Stepanov, A. V. 1975, Issled. Geomagn. Aeron. Fiz. Solntsa, 37, 3

Zaitsev, V. V., \& Stepanov, A. V. 1982, Sov. Astron. Lett., 8, 132 